\title{
Analysis of Prognostic Factors Affecting Short-term and Long-term Outcomes of Gastric Cancer Resection
}

\author{
PIOTR KULIG ${ }^{1,2}$, PRZEMYSŁAW NOWAKOWSKI ${ }^{1}$, MAREK SIERZEGA ${ }^{3}$, RADOSŁAW PACH $^{3}$, \\ OLIWIA MAJEWSKA ${ }^{2}$, ANNA MARKIEWICZ ${ }^{4}$, PIOTR KOŁODZIEJCZYK ${ }^{3}$, JAN KULIG ${ }^{3}$ and PIOTR RICHTER ${ }^{3}$ \\ ${ }^{1}$ Department of Vascular Surgery, American Heart of Poland, Chrzanow, Poland; \\ ${ }^{2}$ Andrzej Frycz Modrzewski Medical Krakow University, Krakow, Poland; \\ ${ }^{3}$ Department of General, Oncological and Gastrointestinal Surgery, \\ Jagiellonian University Medical College, Krakow, Poland; \\ ${ }^{4}$ Department of Ophthalmology and Ocular Oncology, Jagiellonian University Medical College, Krakow, Poland
}

\begin{abstract}
Background: The aim of this study was the analysis of the influence of prognostic factors on short- and long-term outcomes of gastric cancer resection. Patients and Methods: A database of 709 patients who had gastric cancer resection between 2007 and 2015 was compiled. Results: Total gastrectomy (TG) and subtotal proximal gastrectomy (SPG) significantly increased the risk of overall complications ( $p=0.0015$ and 0.0173 , respectively) and surgical complications ( $p=0.0141$ and 0.0035 , respectively). Moreover the resection of an additional organ was an independent prognostic factor of overall complications $(p<0.0001)$, systemic complications $(p=0.0503)$, surgical complications $(p<0.0001)$ and relaparotomy $(p=0.0259) . T$ stage $(p<0.0001), N$ stage $(p<0.0001), M$ stage $(p<0.0001)$ and radical resection $(p<0.0001)$ significantly affected 5 year survival rates. Conclusion: Early diagnosis and radical resection was crucial in 5-year survival rates. However, the type of gastrectomy and the resection of an additional organ were the most important factors in short-term outcomes of treatment for such patients.
\end{abstract}

Gastric cancer is the second most common cancer of the gastrointestinal tract in the world. In 2020, the incidence of new cases of gastric cancer was estimated at $1,089,103$, with a mortality rate of 768,793 people globally. Unlike East Asia, gastric cancer is a relatively rare neoplasm in North

This article is freely accessible online.

Correspondence to: Piotr Kulig, Witkowice Nowe 23, 31-242 Krakow, Poland. Tel: +48660351374 or +48883220224 , e-mail: Kuligos22@interia.pl

Key Words: Gastric cancer resection, prognostic factor, treatment outcome.
America and some highly developed countries in Western Europe. However, even there it is one of the most common causes of death from malignant neoplasms (1). In Poland, the number of deaths from gastric cancer reaches 5000 per year. In 2018, 3155 men and 1745 women died from this in our country (2).

Currently, the majority of publications concern aspects of multimodal therapy with pre- and perioperative chemotherapy, even in the presence of oligometastasis. Nevertheless, surgical resection is still the most effective treatment for such patients, and the principles of surgery of gastric cancer are usually well established (3-5).

Although data from a large number of articles reveals the impact of prognostic factors on the prognosis of patients with gastric cancer resection, survival is significantly improved by the effectiveness of surgical treatment measured by short-term outcomes. Most reports involving prognostic factors for patients after gastric cancer resection focus on the 5-year survival rate. There are no in detail articles on the influence of prognostic factors on short-term outcomes of treatment such as overall complications, systemic complications, surgical complications, relaparotomy and perioperative mortality (6-30). There are many prognostic factors that could affect the short-term outcomes of gastric cancer resection including gender, age, location, histologic type, tumour staging, type of gastrectomy, number of retrieved lymph nodes or resection of an additional organ.

In this study we carried out univariate and multivariate analysis of prognostic factors which, in addition to long-term outcomes (5-year survival), also affected the short-term outcomes of gastric cancer resection. Detailed analysis of prognostic factors made it possible to obtain interesting conclusions about gastric cancer resection, which may significantly influence the optimization of surgical treatment outcomes. 


\section{Patients and Methods}

Patient population. Between 2007 and 2015, 709 patients had gastric cancer resection at the First Department of General, Oncological, Gastrointestinal and Transplantation Surgery, Jagiellonian University Medical College in Krakow. Their database with clinicopathologic features and surgical characteristic was reviewed retrospectively (Table I).

Diagnosis and clinicopathological features. Routine preoperative diagnostics included gastroscopy during which samples were drawn for histopathological examination, chest X-ray, abdominal ultrasonography and, in selected cases, computed tomography. Gastric cancer was defined as a histologically verified primary adenocarcinoma located in the stomach. Patients with other gastric tumors such as gastric lymphoma, gastrointestinal stromal tumor or recurrence of gastric cancer were excluded. The site of the tumor was defined as the location of the main portion of the neoplasm in the upper, middle or lower third of the stomach. Sometimes at diagnosis, gastric cancer was so advanced that occupied two analyzed locations in the stomach (upper/middle/lower), in these cases the location was classified as 'other' (Table I); it was not possible to assume which location was primary. To correctly evaluate the influence of tumor location in the stomach on shortand long-term outcomes of gastric cancer resection in univariate analysis and the logistic regression model, location classified as other was not included. The Lauren classification was used for the histologic evaluation of the tumor (31). The gastric cancer staging system was evaluated by pathological examination of the surgical specimens in accordance with the eighth edition of the TNM staging system of the American Joint Committee on Cancer/Union for International Cancer Control (AJCC/UICC) (32).

Surgical characteristics and treatment. The types of gastrectomy, total/subtotal distal/subtotal proximal and reconstruction options were determined by the location of the tumor, a histopathological examination, and the stage of the disease. However, all resections included in the study were carried out by conventional laparotomy. Lymphadenectomy with the removal of perigastric lymph nodes, lymph nodes around the left gastric artery, common hepatic artery, celiac trunk, splenic artery, hepatoduodenal ligament or additionally paraaortic lymph nodes (D2 or D2+) was standard in cases of radical stomach resections. In some cases, segmental pancreatectomy, spleen and/or bowel resections were performed to obtain potential oncological radicality. Cases with unresectable procedures (gastrojejunostomy, bypass surgery or explorative laparotomy) without gastric cancer resection were excluded from the study. Some patients with advanced gastric cancer (stage II or higher according to TNM classification) received combined therapy using different chemotherapeutic regimens with fluorouracil, cisplatin with fluorouracil, irinotecan with fluorouracil or etoposide, doxorubicin and cisplatin.

Follow-up. Perioperative mortality was defined as any death during the hospital stay after surgery. After discharge, patients had a followed-up every 3-6 months or shorter intervals whenever justifiable. The dates of death were verified using data obtained from the census registry office. All relevant short-term outcomes of treatment, overall complications, systemic complications, surgical complications, relaparotomy as well perioperative mortality were collected and entered into an electronic database (Table II). In some cases, the same patient manifested both systemic and surgical complications. The 5-year survival was understood as long-term outcomes of treatment after gastric cancer resection (Table II). To each of the parameters of short- (overall complications, systemic complications, surgical complications, relaparotomy, perioperative mortality) and long-term (5-year survival) outcomes of treatment was carried out the univariate and multivariate analysis of affecting prognostic factors. These prognostic factors are presented in Table I as clinicopathologic features, and surgical characteristics.

Statistical methods. To determine the relationship between prognostic factors and the outcomes of treatment after gastric cancer resection, we applied the $\chi^{2}$ test and a logistic regression model. The $\chi^{2}$ test was used in the univariate analysis. In view of the available database of the presence of short-term outcomes of treatment and 5-year survival rates, logistic regression models were used in multivariate analysis, which allowed the generation of a dependency model for a binary dependent variable and one or more predictors (prognostic factors). $p<0.05$ was considered statistically significant in two-tailed analysis. The calculations were performed with the statistical package STATISTICA v. 13 and StatsDirect v. 3.3.4.

\section{Results}

Prognostic factors: clinicopathological features and surgical characteristics. The study involved $479(67.6 \%)$ males and 230 $(32.4 \%)$ females. The mean age \pm SD (min-max) was $63.9 \pm 12.0$ (range $=22-89$ ) years, and the incidence of patients older than 70 years was $33.7 \%$. Most tumors were located in the upper third part of the stomach $(31.9 \%)$. The rates of intestinal and diffuse type of Lauren classification were comparable at $43.6 \%$ and $43 \%$, respectively. The proportion of patients with $\mathrm{T}$ stage, $\mathrm{N}$ stage, $\mathrm{M}$ stage according to the eighth edition of TNM (AJCC/UICC) are presented in Table I. The most common type of gastrectomy was total gastrectomy $(64.9 \%)$ and the most common resected additional organ was the spleen $(12.7 \%)$. The incidence of patients who had $>15$ lymph nodes removed was $84.1 \%$. The proportion of patients who underwent radical resection was $59.4 \%$ (Table I).

Outcomes of treatment. Postoperative complications are presented in Table II. The overall percentage of complications was $19.6 \%$, while the rates of systemic and surgical complications were $11.6 \%$ and $11.3 \%$, respectively. The proportion of relaparotomy was $4.8 \%$. The incidence of perioperative mortality was $3.1 \%$. The 5 -year survival rate was $39.1 \%$. Median follow-up was 58.6 (39.5-103.6) months.

Univariate and multivariate analyses. Univariate analysis by the $\chi^{2}$ test revealed potential prognostic factors affecting the short-term outcomes of gastric cancer resection such as overall complications, systemic complications, surgical complications, relaparotomy and perioperative mortality (Table III). The same analysis was used to detect potential prognostic factors of 5year survival for patients (Table IV). Subsequently, prognostic 
Table I. Potential prognostic factors: clinicopathological features and surgical characteristics.

\begin{tabular}{|c|c|}
\hline Prognostic factors & $2007-2015(n=709)$ \\
\hline Clinicopathological features & $\mathrm{n}(\%)$ \\
\hline \multicolumn{2}{|l|}{ Gender } \\
\hline Male & $479(67.6)$ \\
\hline Female & $230(32.4)$ \\
\hline \multicolumn{2}{|l|}{ Age, years } \\
\hline$\leq 70$ & $470(66.3)$ \\
\hline$>70$ & $239(33.7)$ \\
\hline \multicolumn{2}{|l|}{ Location } \\
\hline Upper & $226(31.9)$ \\
\hline Middle & $201(28.3)$ \\
\hline Lower & $148(20.9)$ \\
\hline Other & $134(18.9)$ \\
\hline \multicolumn{2}{|l|}{ Lauren type } \\
\hline Diffuse & $305(43.0)$ \\
\hline Intestinal & 309 (43.6) \\
\hline Mixed & $95(13.4)$ \\
\hline \multicolumn{2}{|c|}{ T stage (eight edition AJCC/UICC) } \\
\hline T1a & $29(4.1)$ \\
\hline $\mathrm{T} 1 \mathrm{~b}$ & $41(5.8)$ \\
\hline $\mathrm{T} 2$ & $124(17.5)$ \\
\hline $\mathrm{T} 3$ & $170(24.0)$ \\
\hline $\mathrm{T} 4 \mathrm{a}$ & $105(14.8)$ \\
\hline $\mathrm{T} 4 \mathrm{~b}$ & $240(33.8)$ \\
\hline \multicolumn{2}{|c|}{$\mathrm{N}$ stage (eight edition AJCC/UICC) } \\
\hline N0 & $111(15.7)$ \\
\hline N1 & $104(14.7)$ \\
\hline N2 & $129(18.2)$ \\
\hline $\mathrm{N} 3 \mathrm{a}$ & $81(11.4)$ \\
\hline $\mathrm{N} 3 \mathrm{~b}$ & $284(40.1)$ \\
\hline \multicolumn{2}{|c|}{ M stage (eight edition AJCC/UICC) } \\
\hline M0 & $423(59.7)$ \\
\hline M1 & $286(40.3)$ \\
\hline Surgical characteristics & $\mathrm{n}(\%)$ \\
\hline \multicolumn{2}{|l|}{ Type of gastrectomy } \\
\hline Total & $526(64.9)$ \\
\hline Subtotal distal & $140(17.3)$ \\
\hline Subtotal proximal & $43(15.3)$ \\
\hline \multicolumn{2}{|l|}{ Resection type } \\
\hline Radical & $421(59.4)$ \\
\hline Palliative & $288(40.6)$ \\
\hline \multicolumn{2}{|c|}{ Number of retrieved lymph nodes } \\
\hline$\leq 15$ & $113(15.9)$ \\
\hline$>15$ & $596(84.1)$ \\
\hline \multicolumn{2}{|c|}{ Resection of an additional organ } \\
\hline None & $577(81.4)$ \\
\hline Spleen & $90(12.7)$ \\
\hline Pancreas & $2(0.3)$ \\
\hline Bowel & $15(2.1)$ \\
\hline Spleen, pancreas & $15(2.1)$ \\
\hline Spleen, bowel & $4(0.6)$ \\
\hline Pancreas, bowel & $2(0.3)$ \\
\hline Spleen, pancreas, bowel & $3(0.4)$ \\
\hline
\end{tabular}

Table II. Outcomes of gastric cancer resection.

\begin{tabular}{lc}
\hline & $2007-2015(\mathrm{n}=709)$ \\
\hline Short-term outcomes of treatment & $\mathrm{n}(\%)$ \\
\hline Overall complications & $139(19.6)$ \\
Systemic complications & $82(11.6)$ \\
Surgical complications & $80(11.3)$ \\
Relapalotomy & $34(4.8)$ \\
Perioperative mortality & $22(3.1)$ \\
\hline Long-term outcomes of treatment & $\mathrm{n}(\%)$ \\
\hline 5-year survival & $277(39.1)$ \\
\hline
\end{tabular}

factors statistically significantly affecting short-term outcomes and 5-year survival in the univariate analysis were assessed with the logistic regression model (Tables V, VI, VII, VIII and IX). Finally, multivariate analysis showed that total gastrectomy [odds ratio $(\mathrm{OR})=1.12,95 \%$ confidence interval $(\mathrm{Cl})=0.93-1.36, p=0.0015]$, and subtotal proximal gastrectomy $(\mathrm{OR}=1.28,95 \% \mathrm{Cl}=1.04-1.58, p=0.0173)$ were independent risk factors of overall complications (Table V). The same types of gastrectomy significantly increased the incidence of surgical complications (total gastrectomy: $\mathrm{OR}=1.19,95 \% \mathrm{Cl}$ $0.94-1.49, \quad p=0.0141 ;$ subtotal proximal gastrectomy: $\mathrm{OR}=1.16 ; 95 \% \mathrm{Cl}=0.91-1.47, p=0.0035) \quad$ (Table VII). Resection of an additional organ significantly increased rates of overall complications $(\mathrm{OR}=2.56,95 \% \mathrm{Cl}=1.79-3.65$, $p<0.0001)$, systemic complications ( $\mathrm{OR}=1.52,95 \% \mathrm{Cl}=0.99$ 2.32, $p=0.0503)$, surgical complications (OR=3.04; $95 \%$ $\mathrm{Cl}=2.07-4.46, p<0.0001)$, and relaparotomy $(\mathrm{OR}=1.9,95 \%$ $\mathrm{Cl}=1.08-3.36, p=0.0259$ ) (Tables V, VI, VII and VIII). The presence of distant metastases (M1 stage) was identified as an independent prognostic factor for a higher perioperative mortality proportion $(\mathrm{OR}=1.36 ; 95 \% \mathrm{Cl}=0.39-4.66, p=0.0422)$ (Table VIII). The multivariate analysis showed that $\mathrm{T}$ stage $(\mathrm{OR}=0.52,95 \% \mathrm{Cl}=0.41-0.66, p<0.0001), \mathrm{N}$ stage $(\mathrm{OR}=0.48$, $95 \% \mathrm{Cl}=0.39-0.6, p<0.0001), \mathrm{M}$ stage $(\mathrm{OR}=0.01,95 \%$ $\mathrm{Cl}=0.001-0.08, p<0.0001)$, location in the middle third part of the stomach $(\mathrm{OR}=1.57,95 \% \mathrm{Cl}=0.73-3.37, p=0,0239)$ and radical resection $(\mathrm{OR}=2.84,95 \% \mathrm{Cl}=2.01-10.95, p<0.0001)$ significantly affected 5-year survival (Table IX).

\section{Discussion}

\section{Clinicopathological features}

Gender. Gastric cancer is twice as common in men as it is in women (33). Gender is reported as being not an independent prognostic factor of survival $(6,7)$. However, in a large database - which is presented in the study Li et al. - white 
Table III. Univariate analysis of clinicopathological features and their influence on the outcomes of gastric cancer resection.

\begin{tabular}{|c|c|c|c|c|c|c|c|c|c|c|c|c|c|}
\hline \multirow[b]{2}{*}{$\begin{array}{l}\text { Clinicopathological } \\
\text { features }\end{array}$} & \multirow[b]{2}{*}{$\mathrm{n}$} & \multicolumn{12}{|c|}{ Outcomes of treatment } \\
\hline & & \multicolumn{2}{|c|}{$\begin{array}{c}\text { Overall } \\
\text { complications }\end{array}$} & \multicolumn{2}{|c|}{$\begin{array}{c}\text { Systemic } \\
\text { complications }\end{array}$} & \multicolumn{2}{|c|}{$\begin{array}{c}\text { Surgical } \\
\text { complications }\end{array}$} & \multicolumn{2}{|c|}{ Relaparotomy } & \multicolumn{2}{|c|}{$\begin{array}{l}\text { Perioperative } \\
\text { mortality }\end{array}$} & \multicolumn{2}{|c|}{$\begin{array}{c}\text { 5-year } \\
\text { survival }\end{array}$} \\
\hline \multicolumn{14}{|l|}{ Gender } \\
\hline Male & 479 & $\begin{array}{c}106 \\
22.1 \%\end{array}$ & $p=0.015^{*}$ & $\begin{array}{c}47 \\
10 \%\end{array}$ & $p=0.008 *$ & $\begin{array}{c}59 \\
12.3 \%\end{array}$ & $p=0.209 *$ & $\begin{array}{c}25 \\
5.2 \%\end{array}$ & $p=0.446^{*}$ & $\begin{array}{c}18 \\
3.8 \%\end{array}$ & $p=0.147^{*}$ & $38 \%$ & $p=0.398^{*}$ \\
\hline Female & 230 & $\begin{array}{c}33 \\
14.4 \%\end{array}$ & & $\begin{array}{c}35 \\
14.6 \%\end{array}$ & & $\begin{array}{c}21 \\
9.1 \%\end{array}$ & & $\begin{array}{c}9 \\
3.9 \%\end{array}$ & & $\begin{array}{c}4 \\
1.7 \%\end{array}$ & & $41.3 \%$ & \\
\hline \multicolumn{14}{|l|}{ Age } \\
\hline$\leq 70$ lat & 470 & $\begin{array}{c}87 \\
18.5 \%\end{array}$ & $p=0.303^{*}$ & $\begin{array}{c}53 \\
9.9 \%\end{array}$ & $p=0.068^{*}$ & $\begin{array}{c}51 \\
10.9 \%\end{array}$ & $p=0.610^{*}$ & $\begin{array}{c}24 \\
5.1 \%\end{array}$ & $p=0.587^{*}$ & $\begin{array}{c}12 \\
2.6 \%\end{array}$ & $p=0.236^{*}$ & $36.6 \%$ & $p=0.058^{*}$ \\
\hline$>70$ lat & 239 & $\begin{array}{c}52 \\
21.8 \%\end{array}$ & & $\begin{array}{c}40 \\
14.7 \%\end{array}$ & & $\begin{array}{c}29 \\
12.1 \%\end{array}$ & & $\begin{array}{c}10 \\
4.2 \%\end{array}$ & & $\begin{array}{c}10 \\
4.2 \%\end{array}$ & & $43.9 \%$ & \\
\hline \multicolumn{14}{|l|}{ Location } \\
\hline Upper & 226 & $\begin{array}{c}49 \\
21.7 \%\end{array}$ & $p=0.593 *$ & $\begin{array}{c}28 \\
12.4 \%\end{array}$ & $p=0.753^{*}$ & $\begin{array}{c}31 \\
13.7 \%\end{array}$ & $p=0.140^{*}$ & $\begin{array}{c}13 \\
5.8 \%\end{array}$ & $p=0.752 *$ & $\begin{array}{c}9 \\
4.0 \%\end{array}$ & $p=0.821^{*}$ & $\begin{array}{c}81 \\
35.8 \%\end{array}$ & $p=0.003^{*}$ \\
\hline Middle & 201 & $\begin{array}{c}38 \\
18.9 \%\end{array}$ & & $\begin{array}{c}20 \\
10.0 \%\end{array}$ & & $\begin{array}{c}24 \\
11.9 \%\end{array}$ & & $\begin{array}{c}9 \\
4.5 \%\end{array}$ & & $\begin{array}{c}5 \\
2.5 \%\end{array}$ & & $\begin{array}{c}87 \\
43.3 \%\end{array}$ & \\
\hline Lower & 148 & $\begin{array}{c}24 \\
16.2 \%\end{array}$ & & $\begin{array}{c}16 \\
10.8 \%\end{array}$ & & $\begin{array}{c}9 \\
6.1 \%\end{array}$ & & $\begin{array}{c}5 \\
3.4 \%\end{array}$ & & $\begin{array}{c}4 \\
2.7 \%\end{array}$ & & $\begin{array}{c}71 \\
48 \%\end{array}$ & \\
\hline \multicolumn{14}{|l|}{ Lauren type } \\
\hline Diffuse & 305 & $\begin{array}{c}56 \\
18.4 \%\end{array}$ & $p=0.065^{*}$ & $\begin{array}{c}40 \\
13.1 \%\end{array}$ & $p=0.194 *$ & $\begin{array}{c}26 \\
8.5 \%\end{array}$ & $p=0.029^{*}$ & $\begin{array}{c}12 \\
3.9 \%\end{array}$ & $p=0.645^{*}$ & $\begin{array}{c}9 \\
3.0 \%\end{array}$ & $p=0.979 *$ & $\begin{array}{c}117 \\
38.4 \%\end{array}$ & $p=0.509^{*}$ \\
\hline Intestinal & 309 & $\begin{array}{c}71 \\
23 \%\end{array}$ & & $\begin{array}{c}36 \\
11.7 \%\end{array}$ & & $\begin{array}{c}46 \\
14.9 \%\end{array}$ & & $\begin{array}{c}17 \\
5.5 \%\end{array}$ & & $\begin{array}{c}10 \\
3.2 \%\end{array}$ & & $\begin{array}{c}127 \\
41.1 \%\end{array}$ & \\
\hline Mixed & 95 & $\begin{array}{c}12 \\
12.6 \%\end{array}$ & & $\begin{array}{c}6 \\
6.3 \%\end{array}$ & & $\begin{array}{c}8 \\
8.4 \%\end{array}$ & & $\begin{array}{c}5 \\
5.3 \%\end{array}$ & & $\begin{array}{c}3 \\
3.2 \%\end{array}$ & & $\begin{array}{c}33 \\
34.7 \%\end{array}$ & \\
\hline \multicolumn{14}{|l|}{ T stage } \\
\hline T1a & 29 & $\begin{array}{c}1 \\
3.5 \%\end{array}$ & $p<0.001^{*}$ & $\begin{array}{c}1 \\
3.5 \%\end{array}$ & $p=0.003^{*}$ & $\begin{array}{c}0 \\
0.0 \%\end{array}$ & $p=0.020^{*}$ & $\begin{array}{c}0 \\
0 \%\end{array}$ & - & $\begin{array}{c}0 \\
0 \%\end{array}$ & - & $\begin{array}{c}28 \\
96.6 \%\end{array}$ & $p<0.001^{*}$ \\
\hline $\mathrm{T} 1 \mathrm{~b}$ & 41 & $\begin{array}{c}2 \\
4.9 \%\end{array}$ & & $\begin{array}{c}1 \\
2.4 \%\end{array}$ & & $\begin{array}{c}1 \\
2.4 \%\end{array}$ & & $\begin{array}{c}0 \\
0 \%\end{array}$ & & $\begin{array}{c}0 \\
0 \%\end{array}$ & & $\begin{array}{c}36 \\
87.8 \%\end{array}$ & \\
\hline $\mathrm{T} 2$ & 124 & $\begin{array}{c}20 \\
6.1 \%\end{array}$ & & $\begin{array}{c}10 \\
8.1 \%\end{array}$ & & $\begin{array}{c}12 \\
9.7 \%\end{array}$ & & $\begin{array}{c}7 \\
5.7 \%\end{array}$ & $p=0.344^{*}$ & $\begin{array}{c}1 \\
0.8 \%\end{array}$ & $p=0.210^{*}$ & $\begin{array}{c}92 \\
74.2 \%\end{array}$ & \\
\hline $\mathrm{T} 3$ & 170 & $\begin{array}{c}25 \\
14.7 \%\end{array}$ & & $\begin{array}{c}14 \\
8.2 \%\end{array}$ & & $\begin{array}{c}15 \\
8.8 \%\end{array}$ & & $\begin{array}{c}6 \\
3.5 \%\end{array}$ & & $\begin{array}{c}5 \\
2.9 \%\end{array}$ & & $\begin{array}{c}84 \\
49.4 \%\end{array}$ & \\
\hline $\mathrm{T} 4 \mathrm{a}$ & 105 & $\begin{array}{c}26 \\
24.8 \%\end{array}$ & & $\begin{array}{c}13 \\
12.4 \%\end{array}$ & & $\begin{array}{c}17 \\
16.2 \%\end{array}$ & & $\begin{array}{c}6 \\
5.7 \%\end{array}$ & & $\begin{array}{c}5 \\
4.8 \%\end{array}$ & & $\begin{array}{c}21 \\
20.0 \%\end{array}$ & \\
\hline $\mathrm{T} 4 \mathrm{~b}$ & 240 & $\begin{array}{c}65 \\
27.1 \%\end{array}$ & & $\begin{array}{c}43 \\
17.9 \%\end{array}$ & & $\begin{array}{c}35 \\
14.6 \%\end{array}$ & & $\begin{array}{c}15 \\
6.3 \%\end{array}$ & & $\begin{array}{c}11 \\
4.6 \%\end{array}$ & & $\begin{array}{c}36 \\
87.8 \%\end{array}$ & \\
\hline \multicolumn{14}{|l|}{$\mathrm{N}$ stage } \\
\hline No & 111 & $\begin{array}{c}8 \\
7.2 \%\end{array}$ & $p<0.001^{*}$ & $\begin{array}{c}4 \\
3.6 \%\end{array}$ & $p<0.001^{*}$ & $\begin{array}{c}5 \\
4.5 \%\end{array}$ & $p=0.029 *$ & $\begin{array}{c}4 \\
3.6 \%\end{array}$ & $p=0.282^{*}$ & $\begin{array}{c}1 \\
0.9 \%\end{array}$ & $p=0.242 *$ & $\begin{array}{c}107 \\
96.4 \%\end{array}$ & $p<0.001^{*}$ \\
\hline N1 & 104 & $\begin{array}{c}14 \\
13.5 \%\end{array}$ & & $\begin{array}{c}8 \\
7.7 \%\end{array}$ & & $\begin{array}{c}8 \\
7.7 \%\end{array}$ & & $\begin{array}{c}3 \\
2.9 \%\end{array}$ & & $\begin{array}{c}1 \\
1.0 \%\end{array}$ & & $\begin{array}{c}87 \\
83.7 \%\end{array}$ & \\
\hline N2 & 129 & $\begin{array}{c}19 \\
14.7 \%\end{array}$ & & $\begin{array}{c}7 \\
5.4 \%\end{array}$ & & $\begin{array}{c}14 \\
10.9 \%\end{array}$ & & $\begin{array}{c}4 \\
3.1 \%\end{array}$ & & $\begin{array}{c}4 \\
3.1 \%\end{array}$ & & $\begin{array}{c}45 \\
34.9 \%\end{array}$ & \\
\hline N3a & 81 & $\begin{array}{c}16 \\
19.8 \%\end{array}$ & & $\begin{array}{c}10 \\
12.4 \%\end{array}$ & & $\begin{array}{c}10 \\
12.4 \%\end{array}$ & & $\begin{array}{c}7 \\
8.6 \%\end{array}$ & & $\begin{array}{c}4 \\
4.9 \%\end{array}$ & & $\begin{array}{c}15 \\
18,5 \%\end{array}$ & \\
\hline $\mathrm{N} 3 \mathrm{~b}$ & 284 & $\begin{array}{c}82 \\
28.9 \%\end{array}$ & & $\begin{array}{c}53 \\
18.7 \%\end{array}$ & & $\begin{array}{c}43 \\
15.1 \%\end{array}$ & & $\begin{array}{c}16 \\
5.6 \%\end{array}$ & & $\begin{array}{c}12 \\
4.2 \%\end{array}$ & & $\begin{array}{c}23 \\
8.1 \%\end{array}$ & \\
\hline M stage & & & & & & & & & & & & & \\
\hline M0 & 423 & $\begin{array}{c}60 \\
14.2 \%\end{array}$ & $p<0.001^{*}$ & $\begin{array}{c}32 \\
7.6 \%\end{array}$ & $p<0.001 *$ & $\begin{array}{c}36 \\
8.5 \%\end{array}$ & $p=0.005^{*}$ & $\begin{array}{c}16 \\
3.8 \%\end{array}$ & $p=0.125^{*}$ & $\begin{array}{c}8 \\
1.9 \%\end{array}$ & $p=0.024 *$ & $\begin{array}{c}276 \\
65.3 \%\end{array}$ & $p<0.001^{*}$ \\
\hline M1 & 286 & $\begin{array}{c}79 \\
27.6 \% \\
27.8 \%\end{array}$ & & $\begin{array}{c}50 \\
17.5 \% \\
17.4 \%\end{array}$ & & $\begin{array}{c}44 \\
15.4 \% \\
15.6 \%\end{array}$ & & $\begin{array}{c}18 \\
6.3 \% \\
6.6 \%\end{array}$ & & $\begin{array}{c}14 \\
4.9 \% \\
4.9 \%\end{array}$ & & $\begin{array}{c}1 \\
0.4 \% \\
0.4 \%\end{array}$ & \\
\hline
\end{tabular}

$* \chi^{2}$ test. 
Table IV. Univariate analysis of surgical characteristics on the outcomes of gastric cancer resection.

\begin{tabular}{|c|c|c|c|c|c|c|c|c|c|c|c|c|c|}
\hline \multirow[b]{2}{*}{$\begin{array}{l}\text { Surgical } \\
\text { characteristics }\end{array}$} & \multirow[b]{2}{*}{$\mathrm{N}$} & \multicolumn{12}{|c|}{ Outcomes of treatment } \\
\hline & & \multicolumn{2}{|c|}{$\begin{array}{c}\text { Overall } \\
\text { complications }\end{array}$} & \multicolumn{2}{|c|}{$\begin{array}{c}\text { Systemic } \\
\text { complications }\end{array}$} & \multicolumn{2}{|c|}{$\begin{array}{c}\text { Surgical } \\
\text { complications }\end{array}$} & \multicolumn{2}{|c|}{ Relaparotomy } & \multicolumn{2}{|c|}{$\begin{array}{c}\text { Perioperative } \\
\text { mortality }\end{array}$} & \multicolumn{2}{|c|}{$\begin{array}{l}\text { 5-year } \\
\text { survival }\end{array}$} \\
\hline \multicolumn{14}{|l|}{ Type of gastrectomy } \\
\hline Total & 526 & $\begin{array}{c}115 \\
21.9 \%\end{array}$ & $p=0.031^{*}$ & $\begin{array}{c}68 \\
12.9 \%\end{array}$ & $p=0.146^{*}$ & $\begin{array}{c}67 \\
12.7 \%\end{array}$ & $p=0.031 *$ & $\begin{array}{c}27 \\
5.1 \%\end{array}$ & $p=0.421^{*}$ & $\begin{array}{c}20 \\
3.8 \%\end{array}$ & $p=0.058^{*}$ & $\begin{array}{c}186 \\
35.4 \%\end{array}$ & $p=0.001^{*}$ \\
\hline Subtotal distal & 140 & $\begin{array}{c}17 \\
12.1 \%\end{array}$ & & $\begin{array}{l}10 \\
7.1 \%\end{array}$ & & $\begin{array}{c}7 \\
5 \%\end{array}$ & & $\begin{array}{c}4 \\
2.9 \%\end{array}$ & & $\begin{array}{c}0 \\
0.0 \%\end{array}$ & & $\begin{array}{c}74 \\
52.9 \%\end{array}$ & \\
\hline Subtotal proximal & 43 & $\begin{array}{c}7 \\
16.3 \%\end{array}$ & & $\begin{array}{c}4 \\
9.3 \%\end{array}$ & & $\begin{array}{c}6 \\
14.0 \%\end{array}$ & & $\begin{array}{c}3 \\
7,0 \%\end{array}$ & & $\begin{array}{c}2 \\
4,7 \%\end{array}$ & & $\begin{array}{c}17 \\
39,5 \%\end{array}$ & \\
\hline \multicolumn{14}{|l|}{$\begin{array}{l}\text { Resection of an } \\
\text { additional organ }\end{array}$} \\
\hline No & 577 & $\begin{array}{c}85 \\
14.7 \%\end{array}$ & $p<0.001 *$ & $\begin{array}{c}56 \\
9.7 \%\end{array}$ & $p=0.002 *$ & $\begin{array}{c}42 \\
7.3 \%\end{array}$ & $p<0.001 *$ & $\begin{array}{c}22 \\
3.8 \%\end{array}$ & $p=0.008^{*}$ & $\begin{array}{c}14 \\
2.4 \%\end{array}$ & $p=0.015^{*}$ & $\begin{array}{c}246 \\
42.6 \%\end{array}$ & $p<0.001 *$ \\
\hline 1 & 107 & $\begin{array}{c}40 \\
37.4 \%\end{array}$ & & $\begin{array}{c}19 \\
17.8 \%\end{array}$ & & $\begin{array}{c}28 \\
26.2 \%\end{array}$ & & $\begin{array}{c}8 \\
7.5 \%\end{array}$ & & $\begin{array}{c}5 \\
4.7 \%\end{array}$ & & $\begin{array}{c}30 \\
28 \%\end{array}$ & \\
\hline$>1$ & 25 & $\begin{array}{c}14 \\
56.0 \%\end{array}$ & & $\begin{array}{c}7 \\
28.0 \%\end{array}$ & & $\begin{array}{c}10 \\
40.0 \%\end{array}$ & & $\begin{array}{c}4 \\
16.0 \%\end{array}$ & & $\begin{array}{c}3 \\
12.0 \%\end{array}$ & & $\begin{array}{c}1 \\
4.0 \%\end{array}$ & \\
\hline \multicolumn{14}{|l|}{$\begin{array}{l}\text { Resection of an } \\
\text { additional organ }\end{array}$} \\
\hline No & 577 & $\begin{array}{c}85 \\
14.7 \%\end{array}$ & $p<0.001 *$ & $\begin{array}{c}56 \\
9.7 \%\end{array}$ & $p=0.020^{*}$ & $\begin{array}{c}42 \\
7.3 \%\end{array}$ & $p<0.001 *$ & $\begin{array}{c}22 \\
3,8 \%\end{array}$ & $p<0.001^{*}$ & $\begin{array}{c}14 \\
2.4 \%\end{array}$ & $p=0.045^{*}$ & $\begin{array}{c}246 \\
42.6 \%\end{array}$ & $p=0.001 *$ \\
\hline Spleen & 90 & $\begin{array}{c}35 \\
38.9 \%\end{array}$ & & $\begin{array}{c}16 \\
17.8 \%\end{array}$ & & $\begin{array}{c}26 \\
28.9 \%\end{array}$ & & $\begin{array}{c}8 \\
8.9 \%\end{array}$ & & $\begin{array}{c}4 \\
4.4 \%\end{array}$ & & $\begin{array}{c}28 \\
31.1 \%\end{array}$ & \\
\hline Bowel & 15 & $\begin{array}{c}3 \\
20 \%\end{array}$ & & $\begin{array}{c}2 \\
13.3 \%\end{array}$ & & $\begin{array}{c}1 \\
6.7 \%\end{array}$ & & $\begin{array}{c}0 \\
0 \%\end{array}$ & & $\begin{array}{c}1 \\
6.7 \%\end{array}$ & & $\begin{array}{c}2 \\
13.3 \%\end{array}$ & \\
\hline Pancreas & 2 & $\begin{array}{c}2 \\
100 \%\end{array}$ & & $\begin{array}{c}1 \\
50 \%\end{array}$ & & $\begin{array}{c}1 \\
50 \%\end{array}$ & & $\begin{array}{c}0 \\
0 \%\end{array}$ & & $\begin{array}{c}0 \\
0 \%\end{array}$ & & $\begin{array}{c}0 \\
0 \%\end{array}$ & \\
\hline Spleen, bowel & 4 & $\begin{array}{c}3 \\
75 \%\end{array}$ & & $\begin{array}{c}1 \\
25 \%\end{array}$ & & $\begin{array}{c}2 \\
50 \%\end{array}$ & & $\begin{array}{c}1 \\
25 \%\end{array}$ & & $\begin{array}{c}0 \\
0 \%\end{array}$ & & $\begin{array}{c}0 \\
0 \%\end{array}$ & \\
\hline Spleen, pancreas & 15 & $\begin{array}{c}6 \\
40 \%\end{array}$ & & $\begin{array}{c}5 \\
29.4 \%\end{array}$ & & $\begin{array}{c}5 \\
29.4 \%\end{array}$ & & $\begin{array}{c}1 \\
5.9 \%\end{array}$ & & $\begin{array}{c}2 \\
11.8 \%\end{array}$ & & $\begin{array}{c}1 \\
5.9 \%\end{array}$ & \\
\hline Pancreas, bowel & 2 & $\begin{array}{c}2 \\
100 \%\end{array}$ & & $\begin{array}{c}1 \\
50 \%\end{array}$ & & $\begin{array}{c}1 \\
50 \%\end{array}$ & & $\begin{array}{c}0 \\
0 \%\end{array}$ & & $\begin{array}{c}0 \\
0 \%\end{array}$ & & $\begin{array}{c}0 \\
0 \%\end{array}$ & \\
\hline $\begin{array}{l}\text { Spleen, pancreas, } \\
\text { bowel }\end{array}$ & 4 & $\begin{array}{c}3 \\
75 \%\end{array}$ & & $\begin{array}{c}1 \\
25 \%\end{array}$ & & $\begin{array}{c}3 \\
75 \%\end{array}$ & & $\begin{array}{c}2 \\
50 \%\end{array}$ & & $\begin{array}{c}1 \\
25 \%\end{array}$ & & $0 \%$ & \\
\hline $\begin{array}{l}\text { Number of retrieved } \\
\text { lymph nodes }\end{array}$ & & & & & & & & & & & & & \\
\hline$>15$ & 596 & $\begin{array}{c}114 \\
19.1 \%\end{array}$ & $p=0.462^{*}$ & $\begin{array}{c}66 \\
11.1 \%\end{array}$ & $p=0.347^{*}$ & $\begin{array}{c}65 \\
10.9 \%\end{array}$ & $p=0.466^{*}$ & $\begin{array}{c}28 \\
4.7 \%\end{array}$ & $p=0.780^{*}$ & $\begin{array}{c}17 \\
2.9 \%\end{array}$ & $p=0.377^{*}$ & $\begin{array}{c}247 \\
41.4 \%\end{array}$ & $p=0.003^{*}$ \\
\hline$\leq 15$ & 113 & $\begin{array}{c}25 \\
22.1 \%\end{array}$ & & $\begin{array}{c}16 \\
14.2 \%\end{array}$ & & $\begin{array}{c}15 \\
13.3 \%\end{array}$ & & $\begin{array}{c}6 \\
5.3 \%\end{array}$ & & $\begin{array}{c}5 \\
4.4 \%\end{array}$ & & $\begin{array}{c}30 \\
26.6 \%\end{array}$ & \\
\hline Resection type & & & & & & & & & & & & & \\
\hline Radical & 421 & $\begin{array}{c}61 \\
14.5 \%\end{array}$ & $p<0.001^{*}$ & $\begin{array}{c}32 \\
7.6 \%\end{array}$ & $p<0.001 *$ & $\begin{array}{c}38 \\
9.0 \%\end{array}$ & $p=0.022 *$ & $\begin{array}{c}17 \\
4.0 \%\end{array}$ & $p=0.254^{*}$ & $\begin{array}{c}9 \\
2.1 \%\end{array}$ & $p=0.073^{*}$ & $\begin{array}{c}277 \\
65.8 \%\end{array}$ & $p<0.001^{*}$ \\
\hline Paliative & 288 & $\begin{array}{c}7 \\
27.1 \%\end{array}$ & & $\begin{array}{c}50 \\
17.4 \%\end{array}$ & & $\begin{array}{c}42 \\
14.6 \%\end{array}$ & & $\begin{array}{c}17 \\
5.9 \%\end{array}$ & & $\begin{array}{c}13 \\
4.5 \%\end{array}$ & & $\begin{array}{c}0 \\
0 \%\end{array}$ & \\
\hline
\end{tabular}

$* \chi^{2}$ test.

female patients as well as male Asian patients showed an independent prognostic factor for better survival in gastric cancer. According to the same database, the prognosis among black female patients between 1973 and 2003 was better than that of men, whereas sex-related survival was not significant in the black race between 2004 and 2013 (8). In Poland, epidemiological data on gastric cancer has stabilized in the last few years $(5,34,35)$. Multivariate analysis in our study showed that gender was not a significant prognostic factor that affected overall complications, systemic complications, surgical complications, relaparotomy as well perioperative mortality and 5-year survival. 
Table V. The logistic regression model for overall complications.

\begin{tabular}{lllcr}
\hline Prognostic factors & OR & $95 \%$ CI & Coefficient & $p$-Value \\
\hline Intercept) & n/a & & -2.8 & $<0.0001$ \\
Gender/male & 1.57 & $0.99-2.5$ & 0.45 & 0.0555 \\
T stage & 1.05 & $0.82-1.36$ & 0.05 & 0.6733 \\
N stage & 1.15 & $0.8-1.65$ & 0.14 & 0.4458 \\
M stage & 0.87 & $0.22-3.42$ & & 0.8394 \\
Type of & & & 0.12 & 0.0015 \\
Gstrectomy & & & 1.06 & 0.2258 \\
$\quad$ Total & 1.12 & $0.93-1.36$ & 0.25 & 0.0173 \\
$\quad$ Subtotal distal & 0.34 & $0.18-0.66$ & 0.94 & $<0.0001$ \\
$\quad$ Subtotal proximal & 1.28 & $1.04-1.58$ & 0.001 & 0.0989 \\
Resection of & & & & \\
an additional organ & 2.56 & $0.27-3.74$ & & \\
Resection type/radical & 1.001 & & & \\
\hline
\end{tabular}

OR: Odds ratio; CI: confidence interval.

Table VI. The logistic regression model for systemic complications.

\begin{tabular}{|c|c|c|c|c|}
\hline Prognostic factors & OR & $95 \% \mathrm{CI}$ & Coefficient & $p$-Value \\
\hline (Intercept) & $\mathrm{n} / \mathrm{a}$ & & -3.7 & $<0.0001$ \\
\hline Gender/male & 1.98 & $1.09-3.6$ & 0.68 & 0.2548 \\
\hline $\mathrm{T}$ stage & 1.027 & $1.005-1.05$ & 0.03 & 0.1527 \\
\hline $\mathrm{N}$ stage & 0.25 & $0.092-0.68$ & 1.38 & 0.6815 \\
\hline M stage & 0.82 & $0.17-3.99$ & 0.2 & 0.8045 \\
\hline Resection of an additional organ & 1.52 & $0.99-2.32$ & 0.42 & 0.0503 \\
\hline Resection type/radical & 0.76 & $1.17-3.38$ & 0.28 & 0.7156 \\
\hline
\end{tabular}

OR: Odds ratio; CI: confidence interval.

Age. The incidence of gastric cancer increases with age and most often occurs in the $7^{\text {th }}$ decade of life (33). It is usually acknowledged that in gastric cancer surgery older patients demonstrate increased risk of complications and a poorer prognosis $(6,7,9-13)$. Although most articles on the impact of age on prognosis of patients with gastric cancer recognize old age as more than 60 years, the $7^{\text {th }}$ decade of life is usually a period of relatively good health nowadays, therefore, in our study, the border age affecting the outcomes of treatment was fixed at 70 years $(6,7,10,11)$. In the present study, the logistic regression model revealed that even at an age $>70$, this was not an independent prognostic factor influencing the short- and long-term outcomes of treatment for patients with gastric cancer resections. Similarly, Kulig et al. did not find differences in complications and perioperative mortality rates, except for a higher incidence of cardiopulmonary complications in older patients undergoing gastric cancer resection $(6.6 \% \mathrm{vs}$. $12.3 \%)$. The median survival of patients was not significantly longer in younger (30.8 months) vs. older (24.1 months) patients $(p=0.056)(14)$. Another report from Poland stratified patients into four groups according to their age: 2950 years (group I), 51-65 years (group II), 66-75 years (group III) and 76-92 years (group IV). The middle-aged patients (group II) had significantly better 3-year survival than either the youngest (group I) or the oldest patients (group IV) (15). Nakamura et al. reported that youth is a prognostic factor of better survival for early gastric cancer, however for advanced gastric cancer, younger patients are prone to more advanced stages of TNM and a poorer prognosis (36). Saito et al. concluded that elderly patients undergo less aggressive surgical resections such as extended lymph node excision or multi-organ resections and present a poorer prognosis (37).

Tumor location. Incidence of gastric cancer situated in the lower or middle third part of the stomach have been steadily going down. Incidence in the upper third part is stable or higher, which could be caused by an inappropriate diet, or obesity and reflux disease in patients with gastric cancer (1721). However, the occurrence of this prognostic factor depends on geographical location and race, for example, in 
Table VII. The logistic regression model for surgical complications.

\begin{tabular}{|c|c|c|c|c|}
\hline Prognostic factors & OR & $95 \% \mathrm{CI}$ & Coefficient & $p$-Value \\
\hline (Intercept) & $\mathrm{n} / \mathrm{a}$ & & -2.76 & 0.0003 \\
\hline \multicolumn{5}{|l|}{ Type of gastrectomy } \\
\hline Total & 1.19 & $0.94-1.49$ & 0.17 & 0.0141 \\
\hline Subtotal distal & 0.28 & $0.12-0.66$ & 1.26 & 0.2391 \\
\hline Subtotal proximal & 1.16 & $0.91-1.47$ & 0.14 & 0.0035 \\
\hline \multicolumn{5}{|l|}{ Lauren type } \\
\hline Diffuse & 0.29 & $0.16-0.57$ & 1.21 & 0.2972 \\
\hline Intestinal & 0.67 & $0.36-1.24$ & 0.4 & 0.2016 \\
\hline Mixed & 0.31 & $0.15-0.67$ & 1.15 & 0.2914 \\
\hline T stage & 1.13 & $0.89-1.44$ & 0.12 & 0.3044 \\
\hline $\mathrm{N}$ stage & 1.13 & $0.8-1.45$ & 0.12 & 0.3584 \\
\hline M stage & 1.73 & $0.31-9.67$ & 0.55 & 0.5314 \\
\hline \multicolumn{5}{|l|}{ Resection of } \\
\hline an additional organ & 3.04 & $2.07-4.46$ & 1.11 & $<0.0001$ \\
\hline Resection type/radical & 1.16 & $0.92-1.47$ & 0.15 & 0.1947 \\
\hline
\end{tabular}

OR: Odds ratio; CI: confidence interval.

Table VIII. The logistic regression model for relaparotomy and perioperative mortality.

\begin{tabular}{|c|c|c|c|c|}
\hline Prognostic factors & OR & $95 \% \mathrm{CI}$ & Coefficient & $p$-Value \\
\hline (Intercept) & $\mathrm{n} / \mathrm{a}$ & & -2.17 & 0.0296 \\
\hline Resection of an additional organ & 1.9 & $1.08-3.36$ & 0.64 & 0.0259 \\
\hline \multirow[t]{2}{*}{ Prognostic factors } & \multicolumn{4}{|c|}{ The logistic regression model for perioperative moratlity } \\
\hline & OR & $95 \% \mathrm{CI}$ & Coefficient & $p$-Value \\
\hline (intercept) & $\mathrm{n} / \mathrm{a}$ & & -2.17 & 0.0066 \\
\hline M stage & 1.36 & $0.39-4.66$ & 0.31 & 0.0422 \\
\hline Resection of an additional organ & 4.37 & $0.62-31.06$ & 1.48 & 0.1399 \\
\hline
\end{tabular}

OR: Odds ratio; CI: confidence interval.

Table IX. The logistic regression model for 5-year survival.

\begin{tabular}{|c|c|c|c|c|}
\hline Prognostic factors & OR & $95 \% \mathrm{CI}$ & Coefficient & $p$-Value \\
\hline (intercept) & $\mathrm{n} / \mathrm{a}$ & & -0.15 & 0.1084 \\
\hline Resection type/radical & 2.84 & $2.01-10.95$ & 6.55 & $<0.0001$ \\
\hline \multicolumn{5}{|l|}{ Type of gastrectomy } \\
\hline Total & 0.76 & $0.61-0.96$ & 0.27 & 0.1891 \\
\hline Subtotal distal & 0.28 & $0.08-1.046$ & 1.26 & 0.5842 \\
\hline Subtotal proximal & 0.855 & $0.53-1.37$ & 0.15 & 0.5198 \\
\hline Resection of an additional organ & 0.47 & $0.32-0.69$ & 0.19 & 0.5373 \\
\hline$>15$ retrieved lymph nodes & 0.83 & $0.34-2.05$ & 0.18 & 0.691 \\
\hline \multicolumn{5}{|l|}{ Location } \\
\hline Upper & 0.97 & $0.47-2.02$ & 0.02 & 0.945 \\
\hline Middle & 1.57 & $0.73-3.37$ & 0.46 & 0.0239 \\
\hline Lower & 1.22 & $0.52-2.84$ & 0.2 & 0.0541 \\
\hline T stage & 0.52 & $0.41-0.66$ & 0.64 & $<0.0001$ \\
\hline $\mathrm{N}$ stage & 0.48 & $0.39-0.6$ & 0.71 & $<0.0001$ \\
\hline M stage & 0.01 & $0.001-0.08$ & 4.42 & $<0.0001$ \\
\hline
\end{tabular}

OR: Odds ratio; CI: confidence interval. 
Asia the proportion of tumors in the lower third part of the stomach exceeds $50 \%$ (6).

Tan et al., Zhao et al., and Lee at al. indicated that the location in the upper third part of the stomach was not an independent prognostic factor of poor survival $(6,25,26)$. In this study, the most common location was the upper third part of the stomach $(31.9 \%)$. In multivariate analysis, location was not a prognostic factor that affected overall complications, systemic and surgical complications, relaparotomy as well perioperative mortality, but the location in the middle third part of the stomach had influence on improved 5-year survival rates $(p=0.0239)$. Conclusions from most reports are consistent with the results of our study. Petrelli et al. reviewed 50 studies including 128,268 patients with gastric cancer, they indicated that a location in the upper third part of the stomach entailed increased risk of tumor-related death and was an important prognostic factor of poorer prognosis (22). Matsuda et al. and $\mathrm{Yu}$ et al. Also found the same, i.e. that patients who underwent resection for gastric cancer with proximal cancer had a poorer 5-year survival $(23,24)$.

Lauren types and tumor staging. In line with the results of our study, the proportion of intestinal type gastric cancers has decreased, while the incidence of diffuse type has recently increased (38-42). Tan et al. noticed that the histological type in gastric cancer patients does not effect prognosis (6). However, most reports have shown that patients with intestinal type gastric cancer demonstrate significantly better survival than diffuse type and mixed type (27-29). In our study, the Lauren type was not an independent prognostic factor that affected the short-term outcomes of treatment and the 5-year survival rates.

As we all know, early stages (according to TNM classification) have an influence on better prognosis after gastric cancer resection $(6,7,10,25,26,30)$. Although in studies from the Netherlands and France where stage IV remained stable over the last few years, the staging of TNM of gastric cancer at diagnosis significantly decreased over the last decades $(38,43-47)$. In the present study, early gastric cancer occurred in $9.9 \%$ of cases, while the proportion of patients with stage IV cancer compromised $40.3 \%$. In multivariate analysis, T stage $(p<0.0001), \mathrm{N}$ stage $(p<0.0001)$ and $\mathrm{M}$ stage $(p<0.0001)$ influenced the 5-year survival and the presence of distant metastases (M1 stage) influenced perioperative mortality $(p=0.0422)$. Nevertheless, TNM stages was not independent prognostic factors affecting short-term outcomes of treatment such as overall complications, systemic complications, surgical complications and relaparotomy.

\section{Surgical characteristics}

Type of resection. Some articles reported that the 5-year survival rates for patients with gastric cancer reveal no significant changes depend on type of gastrectomy at the same stage of the disease (48-50). Studies carried out by the Polish Gastric Cancer Study Group have shown that total gastrectomy improves the 5-year survival in patients with stage IIIA according to the TNM classification (51). In multivariate analysis from the present study, the type of gastrectomy (total, subtotal, proximal or subtotal distal) statistically significantly influenced the overall and surgical complications, but not the systemic complications, relaparotomy as well perioperative mortality and 5-year survival rate.

In Asia, the proportion of patients with gastric cancer who undergo radical resection exceeds $90 \%(6,52)$. In this study, this type of resection occurred in $59.4 \%$ of cases. The fact is that opposite to palliative, radical resection is an independent prognostic factor of better survival which is documented in patients in our study $(52,53-55)$. An interesting conclusion from the present study was that, the type of resection (radical, palliative) was not an independent risk factor in the logistic regression model influencing the short-term outcomes of treatment such as overall complications, systemic complications, surgical complications, relaparotomy and perioperative mortality.

Number of retrieved lymph nodes. In the study of Tan et al., the percentage of patients with gastric cancer with the removal of $>15$ lymph nodes was raised from $38.6 \%$ to $81.7 \%$ over a 30 -year period (6). Although some reports indicated higher rates of postoperative complications after extended lymphadenectomy (D2, D2+ or D3) compared to $\mathrm{D} 1$, it is a routine procedure for patients with potentially radical gastric cancer resection, as it raises the oncological radicality and results in better survival for such patients (5658). Marubini et al. did not observe any differences in perioperative mortality depending on the extent of lymphadenectomy (58). In the present study, dissection of $>15$ lymph nodes was a significant prognostic factor of 5year survival in univariate $(p=0.003)$ but not in multivariate analysis $(p=0.691)$. Interestingly, this was not an independent prognostic factor influencing overall, systemic and surgical complications, relaparotomy and perioperative mortality in the logistic regression model.

Resection of an additional organ. Combined resection of the neoplasm-related affected organs such as the spleen, pancreas or bowel, like an extended lymphadenectomy, may cause increased oncological radicality and better survival in patients of gastric cancer resection $(52,53,55,59)$. However, multiple organ resection is associated with relatively high rates of complications and perioperative mortality (53, 59, 60). Furthermore, Mita et al. indicated that extended multi-organ resection could be beneficial only if radical surgery is carried out in such patients (54). Wang et al. reported that patients with locally advanced gastric cancer (T4-TNM) extending to the bowel might benefit from radical resection with 
acceptable rates of postoperative complications (52). Opposite to Wang et al., in multivariate analysis from our study, the resection of an additional organ was an independent risk factor of overall complications $(p<0.0001)$, systemic $(p=0.0503)$ and surgical complications $(p<0.0001)$ as well relaparotomy $(p=0.0259)$, and did not significantly influence 5 -year survival rates $(p=0.5373)$.

Study limitations. A limitation was that this was a retrospective, single-centre research project. The prognostic factors which were chosen for the analysis, for which data in the patient database of the patients was the most complete. Nevertheless, an analysis of all prognostic factors affecting overall complications, systemic and surgical complications, relaparotomy, as well perioperative mortality and 5-year survival of gastric cancer resection exceeds the size of a discussion for a single scientific article.

In fact, conclusions concerning 5-year survival did not differ from the previous papers, regarding the effect of prognostic factors on outcomes of gastric cancer resection. That said, early diagnosis followed by a radical resection is crucial in gastric cancer patients. However, the study database was from recent years (the final follow-up date was December 18, 2020). Moreover, analysis of prognostic factors after gastric cancer resection apart from the 5-year survival details their influence in the short-term treatment outcomes, such as overall complications, systemic and surgical complications, relaparotomy as well as perioperative mortality, which is an added benefit of our study.

\section{Conclusion}

When possible, a subtotal distal gastrectomy should be the preferred type of gastric cancer resection, as it causes significantly less incidence of overall and surgical complications compared to total and subtotal proximal gastrectomy. Resection of an additional organ such as the spleen, pancreas and/or the bowel is not recommended, because of the significantly increased rates of overall complications, systemic and surgical, as well as relaparotomy. In patients with resection of an additional organ, gastric cancer was too advanced in order for this to make a difference in the 5-year survival. Furthermore, the presence of distant metastases significantly increased the proportion of periopertive mortality. A location in the middle third part of the stomach was demonstrated as an independent prognostic factor of improved 5-year survival. However, early recognition with radical resection is still crucial for 5-year survival of patients after gastric cancer resection.

\section{Conflicts of Interest}

The Authors declare that they have no conflicts of interest.

\section{Authors' Contributions}

Piotr Kulig: concept of the study, collection and analysis of patients database, statistical analysis, database results analysis, preparing articles for discussion, writing the manuscript. Przemysław Nowakowski: collection and analysis of patients database, statistical analysis, database results analysis, preparing articles for the discussion. Marek Sierzęga: concept of the study, collection and analysis of patients database, database results analysis, critical review. Radosław Pach: collection and analysis of patients database, statistical analysis, database results analysis. Oliwia Majewska: collection and analysis of patients database, preparing articles for the discussion. Anna Markiewicz: collection and analysis of patients database, statistical analysis, database results analysis. Piotr Kołodziejczyk: concept of the study, database results analysis. Jan Kulig: concept of the study, database results analysis, critical review. Piotr Richter: concept of the study, database results analysis, critical review.

\section{References}

1 Global Cancer Observatory, Stomach Globocan 2020: International Agency for Research on Cancer, World Health Organization. Available at: https://gco.iarc.fr/today/data/ factsheets/cancers/7-Stomach-fact-sheet.pdf [Last accessed on May 26, 2021]

2 Wojciechowska U, Didkowska J, Michałek I, Olasek P and Ciuba A: Nowotwory złośliwe w Polsce w 2018 roku. Krajowy Rejestr Nowotworów, Warszawa pp. 72-73, 2020. Available at: http://onkologia.org.pl/wp-content/uploads/Nowotwory_2018.pdf [Last accessed on May 26, 2021]

3 Beeharry MK, Zhang TQ, Liu WT and Gang ZZ: Optimization of perioperative approaches for advanced and late stages of gastric cancer: clinical proposal based on literature evidence, personal experience, and ongoing trials and research. World J Surg Oncol 18(1): 51, 2020. PMID: 32151257. DOI: 10.1186/s12957-020-01819-6

4 Parisi A, Porzio G and Ficorella C: Multimodality treatment in metastatic gastric cancer: from past to next future. Cancers (Basel) 12(9): 2598, 2020. PMID: 32932914. DOI: 10.3390/cancers 12092598

5 Petrillo A and Smyth EC: Multimodality treatment for localized gastric cancer: state of the art and new insights. Curr Opin Oncol 32(4): 347-355, 2020. PMID: 32541324. DOI: 10.1097/ CCO.0000000000000630

6 Tan YE, Wang PL, Yin SC, Zhang C, Hou WB and Xu HM: Thirty-year trends in clinicopathologic characteristics and prognosis after gastrectomy for gastric cancer: A single institution in Northern China. J Cancer 11(5): 1056-1062, 2020. PMID: 31956352. DOI: 10.7150/jca.36927

7 Alshehri A, Alanezi $\mathrm{H}$ and Kim BS: Prognosis factors of advanced gastric cancer according to sex and age. World J Clin Cases 8(9): 1608-1619, 2020. PMID: 32432139. DOI: 10.12998/wjcc.v8.i9.1608

8 Li H, Wang C, Wei Z, Chen W, Guo Z, He Y and Zhang C: Differences in the prognosis of gastric cancer patients of different sexes and races and the molecular mechanisms involved. Int J Oncol 55(5): 1049-1068, 2019. PMID: 31793655. DOI: $10.3892 /$ ijo.2019.4885 
9 Kim JH, Chin HM and Jun KH: Surgical outcomes and survival after gastrectomy in octogenarians with gastric cancer. J Surg Res 198(1): 80-86, 2015. PMID: 26095423. DOI: 10.1016/ j.jss. 2015.05 .046

10 Talebi A, Mohammadnejad A, Akbari A, Pourhoseingholi MA, Doosti H, Moghimi-Dehkordi B, Agah S and Bahardoust M: Survival analysis in gastric cancer: a multi-center study among Iranian patients. BMC Surg 20(1): 152, 2020. PMID: 32660458. DOI: $10.1186 / \mathrm{s} 12893-020-00816-6$

11 Li Q, Huang LY and Xue HP: Comparison of prognostic factors in different age groups and prognostic significance of neutrophillymphocyte ratio in patients with gastric cancer. World $\mathrm{J}$ Gastrointest Oncol 12(10): 1146-1166, 2020. PMID: 33133383. DOI: $10.4251 /$ wjgo.v12.i10.1146

12 Shibata C, Ogawa H, Nakano T, Koyama K, Yamamoto K, Nagao M, Takeyama D, Takami K, Yasumoto A, Sase T, Kimura SI, Sawada K and Katayose Y: Influence of age on postoperative complications especially pneumonia after gastrectomy for gastric cancer. BMC Surg 19(1): 106, 2019. PMID: 31395044. DOI: 10.1186/s12893-019-0573-x

13 Hsu JT, Liu MS, Wang F, Chang CJ, Hwang TL, Jan YY and Yeh TS: Standard radical gastrectomy in octogenarians and nonagenarians with gastric cancer: are short-term surgical results and long-term survival substantial? J Gastrointest Surg 16(4): 728737, 2012. PMID: 22350724. DOI: 10.1007/s11605-012-1835-4

14 Kulig J, Popiela T, Kolodziejczyk P, Sierzega M, Jedrys J, Szczepanik AM and Polish Gastric Cancer Study Group: Clinicopathological profile and long-term outcome in young adults with gastric cancer: multicenter evaluation of 214 patients. Langenbecks Arch Surg 393(1): 37-43, 2008. PMID: 17618451. DOI: 10.1007/s00423-007-0208-z

15 Ciesielski M, Kruszewski WJ, Szajewski M, Walczak J, Spychalska N, Szefel J and Zieliński J: Extremely high mortality rate after a successful gastrectomy for cancer in older adults. J Gastric Cancer 19(2): 202-211, 2019. PMID: 31245165. DOI: 10.5230/jgc.2019.19.e16

16 Kulig P, Sierzega M, Kowalczyk T, Kolodziejczyk P and Kulig $\mathrm{J}$ : Non-curative gastrectomy for metastatic gastric cancer: rationale and long-term outcome in multicenter settings. Eur $\mathrm{J}$ Surg Oncol 38(6): 490-496, 2012. PMID: 22381671. DOI: $10.1016 /$ j.ejso.2012.01.013

17 Honda M, Wong SL, Healy MA, Nakajima T, Watanabe M, Fukuma S, Fukuhara S and Ayanian JZ: Long-term trends in primary sites of gastric adenocarcinoma in Japan and the United States. J Cancer 8(11): 1935-1942, 2017. PMID: 28819392. DOI: $10.7150 /$ jca. 19174

18 Anderson WF, Camargo MC, Fraumeni JF Jr, Correa P, Rosenberg PS and Rabkin CS: Age-specific trends in incidence of noncardia gastric cancer in US adults. JAMA 303(17): 17231728, 2010. PMID: 20442388. DOI: 10.1001/jama.2010.496

19 Kulig P, Sierzega M, Pach R, Kolodziejczyk P, Kulig J and Polish Gastric Cancer Study Group: Differences in prognosis of Siewert II and III oesophagogastric junction cancers are determined by the baseline tumour staging but not its anatomical location. Eur J Surg Oncol 42(8): 1215-1221, 2016. PMID: 27241921. DOI: $10.1016 /$ j.ejso.2016.04.061

20 Asplund J, Kauppila JH, Mattsson F and Lagergren J: Survival trends in gastric adenocarcinoma: A population-based study in Sweden. Ann Surg Oncol 25(9): 2693-2702, 2018. PMID: 29987609. DOI: 10.1245/s10434-018-6627-y
21 Dassen AE, Dikken JL, Bosscha K, Wouters MW, Cats A, van de Velde CJ, Coebergh JW and Lemmens VE: Gastric cancer: decreasing incidence but stable survival in the Netherlands. Acta Oncol 53(1): 138-142, 2014. PMID: 23829603. DOI: 10.3109/0284186X.2013.789139

22 Petrelli F, Ghidini M, Barni S, Steccanella F, Sgroi G, Passalacqua $\mathrm{R}$ and Tomasello G: Prognostic role of primary tumor location in non-metastatic gastric cancer: a systematic review and meta-analysis of 50 studies. Ann Surg Oncol 24(9): 2655-2668, 2017. PMID: 28299508. DOI: 10.1245/s10434-017$5832-4$

23 Matsuda T, Ohashi M, Tsujiura M, Ida S, Kumagai K, Nunobe S, Sano T and Hiki N: Shorter survival of patients with upperthird gastric cancer preoperatively diagnosed as stage IA compared with those with middle to lower lesions. Ann Surg Oncol 27(1): 276-283, 2020. PMID: 31502022. DOI: 10.1245/s10434-019-07782-1

$24 \mathrm{Yu} \mathrm{X}, \mathrm{Hu} \mathrm{F}$, Li C, Yao Q, Zhang $\mathrm{H}$ and Xue Y: Clinicopathologic characteristics and prognosis of proximal and distal gastric cancer. Onco Targets Ther 11: 1037-1044, 2018. PMID: 29520154. DOI: 10.2147/OTT.S157378

25 Zhao L, Huang H, Zhao D, Wang C, Tian Y, Yuan X, Ma F, Ren H, Zhao Y, Aimaiti S, Zhang S, Zhou H, Wang T, Wang N, Sun Y, Bai X and Chen Y: Clinicopathological characteristics and prognosis of proximal and distal gastric cancer during 19972017 in China National Cancer Center. J Oncol 2019: 9784039, 2019. PMID: 31312217 . DOI: 10.1155/2019/9784039

26 Lee J, Hong Y, Choi Y, Lim H and Lee S: Prognosis of proximal upper-third gastric cancer excluding tumors originating in the esophagogastric junction. Korean Journal of Clinical Oncology 15(2): 93-99, 2020. DOI: 10.14216/kjco.19017

27 Qiu MZ, Cai MY, Zhang DS, Wang ZQ, Wang DS, Li YH and $\mathrm{Xu}$ RH: Clinicopathological characteristics and prognostic analysis of Lauren classification in gastric adenocarcinoma in China. J Transl Med 11: 58, 2013. PMID: 23497313. DOI: 10.1186/1479-5876-11-58

28 Chen YC, Fang WL, Wang RF, Liu CA, Yang MH, Lo SS, Wu CW, Li AF, Shyr YM and Huang KH: Clinicopathological variation of Lauren classification in gastric cancer. Pathol Oncol Res 22(1): 197-202, 2016. PMID: 26502923. DOI: 10.1007/s12253-015-9996-6

29 Qiu M, Zhou Y, Zhang X, Wang Z, Wang F, Shao J, Lu J, Jin Y, Wei X, Zhang D, Wang F, Li Y, Yang D and Xu R: Lauren classification combined with HER2 status is a better prognostic factor in Chinese gastric cancer patients. BMC Cancer 14: 823, 2014. PMID: 25380654. DOI: 10.1186/1471-2407-14-823

30 Yaprak G, Tataroglu D, Dogan B and Pekyurek M: Prognostic factors for survival in patients with gastric cancer: Single-centre experience. North Clin Istanb 7(2): 146-152, 2019. PMID: 32259036. DOI: $10.14744 /$ nci.2019.73549

31 Lauren P: The two histological main types of gastric carcinoma: diffuse and so-called intestinal-type carcinoma. An attempt at a histo-clinical classification. Acta Pathol Microbiol Scand 64: 3149, 1965. PMID: 14320675. DOI: 10.1111/apm.1965.64.1.31

32 Amin MB, Greene FL, Edge SB, Compton CC, Gershenwald JE, Brookland RK, Meyer L, Gress DM, Byrd DR and Winchester DP: The Eighth Edition AJCC Cancer Staging Manual: Continuing to build a bridge from a population-based to a more "personalized" approach to cancer staging. CA Cancer J Clin 67(2): 93-99, 2017. PMID: 28094848. DOI: 10.3322/caac.21388 
33 Bray F, Ferlay J, Soerjomataram I, Siegel RL, Torre LA and Jemal A: Global cancer statistics 2018: GLOBOCAN estimates of incidence and mortality worldwide for 36 cancers in 185 countries. CA Cancer J Clin 68(6): 394-424, 2018. PMID: 30207593. DOI: $10.3322 /$ caac. 21492

34 Didkowska J, Wojciechowska U and Olasek P: Cancer in Poland in 2015. ISSN 0867-8251. Warszawa 2017. National Cancer Registry. Available at: http://onkologia.org.pl/wp-content/uploads/ Nowotwory_2017.pdf [Last accessed on May 26, 2021]

35 Popiela T, Kulig J, Kołodziejczyk P and Sierzega M: Changing patterns of gastric carcinoma over the past two decades in a single institution: clinicopathological findings in 1557 patients. Scand J Gastroenterol 37(5): 561-567, 2002. PMID: 12059058. DOI: $10.1080 / 00365520252903116$

36 Nakamura T, Yao T, Niho $\mathrm{Y}$ and Tsuneyoshi $\mathrm{M}$ : A clinicopathological study in young patients with gastric carcinoma. J Surg Oncol 71(4): 214-219, 1999. PMID: 10440758. DOI: 10.1002/(sici)1096-9098(199908)71:4<214::aid-jso2>3.0.co;2-d

37 Saito H, Osaki T, Murakami D, Sakamoto T, Kanaji S, Tatebe S, Tsujitani $\mathrm{S}$ and Ikeguchi M: Effect of age on prognosis in patients with gastric cancer. ANZ J Surg 76(6): 458-461, 2006. PMID: 16768768. DOI: 10.1111/j.1445-2197.2006.03756.x

38 Yamashita K, Sakuramoto S, Nemoto M, Shibata T, Mieno H, Katada N, Kikuchi S and Watanabe M: Trend in gastric cancer: 35 years of surgical experience in Japan. World J Gastroenterol 17(29): 3390-3397, 2011. PMID: 21876631. DOI: 10.3748/wjg.v 17.i29.3390

39 Levi F, Lucchini F, Negri E, Boyle P and La Vecchia C: Cancer mortality in Europe, 1995-1999, and an overview of trends since 1960. Int J Cancer 110(2): 155-169, 2004. PMID: 15069676. DOI: $10.1002 / \mathrm{ijc} .20097$

$40 \mathrm{Wu}$ H, Rusiecki JA, Zhu K, Potter J and Devesa SS: Stomach carcinoma incidence patterns in the United States by histologic type and anatomic site. Cancer Epidemiol Biomarkers Prev 18(7): 1945-1952, 2009. PMID: 19531677. DOI: 10.1158/10559965.EPI-09-0250

41 Laurén PA and Nevalainen TJ: Epidemiology of intestinal and diffuse types of gastric carcinoma. A time-trend study in Finland with comparison between studies from high- and low-risk areas. Cancer 71(10): 2926-2933, 1993. PMID: 8490820. DOI: $10.1002 / 1097-0142(19930515) 71: 10<2926:$ :aid-cncr282 0711007>3.0.co;2-x

42 Kaneko $\mathrm{S}$ and Yoshimura T: Time trend analysis of gastric cancer incidence in Japan by histological types, 1975-1989. Br J Cancer 84(3): 400-405, 2001. PMID: 11161407. DOI: 10.1054/bjoc. 2000.1602

43 Ahn HS, Lee HJ, Yoo MW, Jeong SH, Park DJ, Kim HH, Kim WH, Lee KU and Yang HK: Changes in clinicopathological features and survival after gastrectomy for gastric cancer over a 20-year period. Br J Surg 98(2): 255-260, 2011. PMID: 21082693. DOI: $10.1002 /$ bjs. 7310

44 Holster IL, Aarts MJ, Tjwa ET, Lemmens VE and Kuipers EJ: Trend breaks in incidence of non-cardia gastric cancer in the Netherlands. Cancer Epidemiol 38(1): 9-15, 2014. PMID: 24309073. DOI: 10.1016/j.canep.2013.11.001

45 Kim HS, Lee H, Jeung HC, Noh SH, Chung HC, Roh JK, Nam $\mathrm{CM}$ and Rha SY: Advanced detection of recent changing trends in gastric cancer survival: up-to-date comparison by period analysis. Jpn J Clin Oncol 41(12): 1344-1350, 2011. PMID: 22128316. DOI: $10.1093 /$ jjco/hyr153
46 Wang W, Zheng C, Fang C, Li P, Xie J, Lin J, Zhan Y, Li W, Chen Y, Sun X, Xu D, Li Y, Huang C and Zhou Z: Time trends of clinicopathologic features and surgical treatment for gastric cancer: Results from 2 high-volume institutions in southern China. Surgery 158(6): 1590-1597, 2015. PMID: 26210225. DOI: $10.1016 /$ j.surg.2015.04.038

47 Herbreteau E, Jooste V, Hamza S, Lepage C, Faivre J and Bouvier AM: Trends in the management of gastric cancer over a 32-year period: a French population-based study. Gastric Cancer 18(1): 129-137, 2015. PMID: 24488016. DOI: 10.1007/s10120-014-0342-6

48 Bozzetti F, Marubini E, Bonfanti G, Miceli R, Piano C and Gennari L: Subtotal versus total gastrectomy for gastric cancer: five-year survival rates in a multicenter randomized Italian trial. Italian Gastrointestinal Tumor Study Group. Ann Surg 230(2): 170-178, 1999. PMID: 10450730. DOI: 10.1097/00000658199908000-00006

49 Ji X, Yan Y, Bu ZD, Li ZY, Wu AW, Zhang LH, Wu XJ, Zong XL, Li SX, Shan F, Jia ZY and Ji JF: The optimal extent of gastrectomy for middle-third gastric cancer: distal subtotal gastrectomy is superior to total gastrectomy in short-term effect without sacrificing long-term survival. BMC Cancer 17(1): 345, 2017. PMID: 28526077. DOI: 10.1186/s12885-017-3343-0

50 Yoo CH, Sohn BH, Han WK and Pae WK: Proximal gastrectomy reconstructed by jejunal pouch interposition for upper third gastric cancer: prospective randomized study. World J Surg 29(12): 1592-1599, 2005. PMID: 16311849. DOI: 10.1007/s00268-005-7793-1

51 Popiela T, Kulig J, Kolodziejcyk P and Polish Gastric Cancer Study Group: [Twenty-year experience with multimodal treatment of gastric cancer patients in Poland]. Zentralbl Chir 126(10): 763-771, 2001. PMID: 11727184. DOI: 10.1055/s2001-18263

52 Wang GC, Liu YJ, Gao CQ, Wang YC, Lv HF, Chen BB, Nie CY, Chen XB and Luo SX: Surgical outcomes and survival for T4 gastric cancer extending to the transverse colon. Ann Transl Med 8(15): 947, 2020. PMID: 32953747. DOI: 10.21037/atm20-3377

53 Carboni F, Lepiane P, Santoro R, Lorusso R, Mancini P, Sperduti I, Carlini $M$ and Santoro E: Extended multiorgan resection for T4 gastric carcinoma: 25-year experience. J Surg Oncol 90(2): 95-100, 2005. PMID: 15844189. DOI: 10.1002/jso.20244

54 Mita K, Ito H, Fukumoto M, Murabayashi R, Koizumi K, Hayashi $\mathrm{T}$ and Kikuchi H: Surgical outcomes and survival after extended multiorgan resection for T4 gastric cancer. Am J Surg 203(1): 107111, 2012. PMID: 21474116. DOI: 10.1016/j.amjsurg.2010.12.007

55 Cheng CT, Tsai CY, Hsu JT, Vinayak R, Liu KH, Yeh CN, Yeh TS, Hwang TL and Jan YY: Aggressive surgical approach for patients with T4 gastric carcinoma: promise or myth? Ann Surg Oncol 18(6): 1606-1614, 2011. PMID: 21222167. DOI: 10.1245/s 10434-010-1534-X

56 Kodera Y, Schwarz RE and Nakao A: Extended lymph node dissection in gastric carcinoma: where do we stand after the Dutch and British randomized trials? J Am Coll Surg 195(6): 855-864, 2002. PMID: 12495318. DOI: 10.1016/s10727515(02)01496-5

57 Karpeh MS, Leon L, Klimstra D and Brennan MF: Lymph node staging in gastric cancer: is location more important than Number? An analysis of 1,038 patients. Ann Surg 232(3): 362-371, 2000. PMID: 10973386. DOI: 10.1097/00000658-200009000-00008 
58 Marubini E, Bozzetti F, Miceli R, Bonfanti G, Gennari L and Gastrointestinal Tumor Study Group: Lymphadenectomy in gastric cancer: prognostic role and therapeutic implications. Eur J Surg Oncol 28(4): 406-412, 2002. PMID: 12099651. DOI: 10.1053/ejso.2001.1247

59 Ozer I, Bostanci EB, Orug T, Ozogul YB, Ulas M, Ercan M, Kece C, Atalay F and Akoglu M: Surgical outcomes and survival after multiorgan resection for locally advanced gastric cancer. Am J Surg 198(1): 25-30, 2009. PMID: 18823618. DOI: 10.1016/j.amjsurg.2008.06.031
60 Kasakura Y, Fujii M, Mochizuki F, Kochi M and Kaiga T: Is there a benefit of pancreaticosplenectomy with gastrectomy for advanced gastric cancer? Am J Surg 179(3): 237-242, 2000. PMID: 10827328. DOI: 10.1016/s0002-9610(00)00293-2

Received April 26, 2021

Revised May 25, 2021

Accepted May 27, 2021 